\title{
THREEFOLDS OF ORDER ONE IN THE SIX-QUADRIC
}

\author{
LEV BORISOV AND JEFF VIACLOVSKY
}

\begin{abstract}
Consider the smooth quadric $Q_{6}$ in $\mathbb{P}^{7}$. The middle homology group $H_{6}\left(Q_{6}, \mathbb{Z}\right)$ is isomorphic to $\mathbb{Z} \oplus \mathbb{Z}$, with a basis given by two classes of linear subspaces. We classify all threefolds of bidegree $(1, p)$ inside $Q_{6}$.
\end{abstract}

\section{INTRODUCTION}

The study of surfaces inside the Grassmannian $G(2,4)$ of lines in the complex projective space of dimension three has a long and rich history, we refer the reader to Gro92 for references. The homology class of any such surface is given by two nonnegative integers $(m, n)$ called the bidegree, with $m$ classically called the order and $n$ called the class. In particular, surfaces of order one have been completely classified, see Ran86 for a modern treatment.

In this paper we are interested in threefolds inside the six-dimensional nonsingular quadric $Q_{6}$ in the complex projective space $\mathbb{P}^{7}$. There are several reasons one may want to study these threefolds. First, the Grassmannian $G(2,4)$ is a nonsingular quadric in $\mathbb{P}^{5}$. The nonsingular quadric $Q_{6}$ is the natural example in the next dimension. Namely, its middle homology $H_{6}\left(Q_{6}, \mathbb{Z}\right)$ is isomorphic to $\mathbb{Z} \oplus \mathbb{Z}$ and it is natural to try to study threefolds inside $Q_{6}$ with a given homology class. We note that it is fairly easy to prove that the only threefold of order zero is a linear $\mathbb{P}^{3}$ of bidegree $(0,1)$, so order one is the first interesting case. Second, the quadric $Q_{6}$ is the orthogonal Grassmannian of isotropic subspaces of dimension four in $\mathbb{C}^{8}$, and thus has special geometric significance. Last, but not least, threefolds of bidegree $(1, p)$ in $Q_{6}$ appear naturally in the study of orthogonal complex structures on subdomains of the sphere $S^{6}$, see [BSV]. This was the original motivation behind our interest in the topic.

The main result of this paper is that every irreducible $Z$ of bidegree $(1, p)$ in $Q_{6}$ is given by one of the following:

- $p=0$ and $Z$ is a horizontal $\mathbb{P}^{3}$.

- $p=1$ and $Z$ is a smooth quadric in $\mathbb{P}^{4} \subset \mathbb{P}^{7}$.

- $p=3$ and $Z$ is a cone over the Veronese surface in $\mathbb{P}^{5} \subset \mathbb{P}^{7}$.

- $p \geq 1$ and $Z$ is a Weil divisor of bidegree $(1, p)$ in a rank four dimension four quadric $Q_{4} \subset Q_{6}$.

Date: August 12, 2008.

Lev Borisov has been partially supported by the National Science Foundation under grant DMS0758480. Jeff Viaclovsky has been partially supported by the National Science Foundation under grant DMS-0804042. 
This classification will also enable us to classify the smooth threefolds of order one; the only examples are the first two cases above, and the Segre embedding for $p=2$, see Theorem 2.10. The plan of the paper is as follows. In Section 2, we state our main results in more detail, and describe the last two cases above more explicitly in terms of homogeneous coordinates on $\mathbb{P}^{7}$. In Section 3 , we collect some preliminary results on the geometry of the quadric $Q_{6}$ and of threefolds $Z \subset Q_{6}$ of bidegree $(1, p)$. In Section 4, we complete the proofs of our main results, Theorems 2.7 and 2.10. Our arguments are completely classical in their nature.

Acknowledgements. We would like to thank Igor Dolgachev for providing useful references Gro92] and [Ran86]. Lev Borisov also thanks Vasilii Iskovskikh for being an excellent undergraduate advisor at Moscow State University in 1987-1992.

\section{Statement of The RESUlts}

Throughout the paper all varieties are considered over the field of complex numbers, and dimension means complex dimension. The main object of our interest is a nonsingular quadric $Q_{6}$ of dimension 6 and rank 8 in the projective space $\mathbb{P}^{7}$. We also think of it as a nondegenerate bilinear form on the corresponding vector space $\mathbb{C}^{8}$, which allows us to define annihilators of projective subspaces of $\mathbb{P}^{7}$.

We recall some standard facts about the integer homology of $Q_{6}$.

Proposition 2.1. The maximum dimension of a linear subspace $\mathbb{P}^{k}$ in $Q_{6}$ is $k=3$. There are two types of such subspaces, which we call horizontal and vertical. The spaces of the same type intersect each other in spaces of odd dimension (empty, $\mathbb{P}^{1}$ or $\left.\mathbb{P}^{3}\right)$ and the spaces of different type intersect each other in spaces of even dimension (point or $\left.\mathbb{P}^{2}\right)$. The homology group $H_{6}\left(Q_{6}, \mathbb{Z}\right)$ is freely generated by the classes of horizontal and vertical subspaces.

Proof. See [GH94, Chapter 6] for the first part of the statement. To show that the vertical and horizontal subspaces generate $H_{6}\left(Q_{6}, \mathbb{Z}\right)$, it suffices to show that the latter is a free abelian group of rank 2 in view of the intersection pairing. One can show this, for example, by realizing $Q_{6}$ as an orthogonal Grassmannian and decomposing it into a union of Schubert cycles.

We will denote by $(a, b)$ the homology class given by $a$ horizontal and $b$ vertical $\mathbb{P}^{3}$-s. The main goal of this paper is to classify subvarieties $Z \subset Q_{6}$ of dimension three and homology class $(1, p)$.

Remark 2.2. For the sake of definitiveness, we can assume that $Q_{6}$ is given by the equation

$$
x_{1} x_{8}-x_{2} x_{7}+x_{3} x_{6}-x_{4} x_{5}=0
$$

in $\mathbb{P}^{7}$ with homogeneous coordinates $\left(x_{1}: \ldots: x_{8}\right)$. In this notation, particular examples of horizontal and vertical subspaces are given by $\left\{x_{4}=x_{6}=x_{7}=x_{8}=0\right\}$ and $\left\{x_{5}=x_{6}=x_{7}=x_{8}=0\right\}$, respectively. Indeed, these subspaces intersect in a $\mathbb{P}^{2}$, so they are of different types, and it is up to us to specify their names. 
We will now explain how one can generate examples of $Z \subset Q_{6}$ of bidegree $(1, p)$. We refer the reader to [Har77, Chapter II] for background on Cartier and Weil divisors.

Proposition 2.3. Consider the dimension four singular quadric $Q_{4}$ of rank four given by the equation $x_{3} x_{6}=x_{4} x_{5}$ in the projective space $\mathbb{P}^{5}$ with homogeneous coordinates $\left(x_{1}: \ldots: x_{6}\right)$. The class group of its Weil divisors is isomorphic to $\mathbb{Z} \oplus \mathbb{Z}$ and is generated by the divisors $D_{1}$ and $D_{2}$ which are cut out by the homogeneous ideals $\left\langle x_{4}, x_{6}\right\rangle$ and $\left\langle x_{5}, x_{6}\right\rangle$ respectively.

Proof. The complement of $D_{1} \cup D_{2}$ in $Q_{4}$ is isomorphic to $\mathbb{C}^{4}$ with coordinates $\left(\frac{x_{1}}{x_{6}}, \frac{x_{2}}{x_{6}}, \frac{x_{4}}{x_{6}}, \frac{x_{5}}{x_{6}}\right)$. Consequently, any divisor on $Q_{4}$ can be pushed away from this $\mathbb{C}^{4}$ and written as a linear combination of $D_{1}$ and $D_{2}$, see also [Har77, Exercise II.6.5].

The quadric $Q_{4}$ is the intersection of the nonsingular quadric $Q_{6}$ given by (2.1) and the dimension five subspace $x_{7}=x_{8}=0$. The rank of the quadratic form drops to 4 , so this $Q_{4}$ is a double cone over a smooth 2-quadric $Q_{2}$.

Proposition 2.4. The images of the classes of $D_{1}$ and $D_{2}$ in $Q_{6}$ under the natural map $H_{6}\left(Q_{4}, \mathbb{Z}\right) \rightarrow H_{6}\left(Q_{6}, \mathbb{Z}\right)$ are given by $(1,0)$ and $(0,1)$, respectively.

Proof. This follows from Remark 2.2 .

The following proposition describes the divisors $D$ of degree $(1, p)$ in $Q_{4}$ for $p \geq 1$. Observe that the divisor $D+(p-1) D_{1}$ is Cartier and is a multiple of the ample divisor $(1,1)$ on $Q_{4}$.

Proposition 2.5. Every Weil divisor $D$ of degree $(1, p)$ on $Q_{4}$ can be written as the divisor of a polynomial $f\left(x_{1}, \ldots, x_{6}\right)$ minus $(p-1) D_{1}$, with the polynomial $f$ of the form

$$
f=g_{p}\left(x_{4}, x_{6}\right)+\sum_{i=1,2,3,5} x_{i} g_{p-1}^{(i)}\left(x_{4}, x_{6}\right)
$$

where $g_{p}$ is homogeneous of degree $p$ and $g_{p-1}^{(i)}$ are homogeneous of degree $p-1$.

Proof. For each $p \geq 0$, we have the short exact sequence of sheaves on $\mathbb{P}^{5}$

$$
0 \rightarrow \mathcal{O}(p-2) \rightarrow \mathcal{O}(p) \rightarrow \mathcal{O}_{Q_{4}}(p, p) \rightarrow 0 .
$$

Since $H^{1}\left(\mathbb{P}^{5}, \mathcal{O}(p-2)\right)=0$ (see [GH94, page 156]), the restriction map

$$
H^{0}\left(\mathbb{P}^{5}, \mathcal{O}(p)\right) \rightarrow H^{0}\left(Q_{4}, \mathcal{O}_{Q_{4}}(p, p)\right)
$$

is surjective. Hence all Cartier divisors of type $(p, p)$ on $Q_{4}$ are given as zeroes of a degree $p$ polynomial $f\left(x_{1}, \ldots, x_{6}\right)$, intersected scheme theoretically with $Q_{4}$.

It remains to investigate the condition on $f$ which says that the corresponding divisor on $Q_{4}$ is of the form $D+(p-1) D_{1}$, that is, that $f$ vanishes to order at least $(p-1)$ on $D_{1}$, modulo the equation of $Q_{4}$. We can use the equation of $Q_{4}$ to assume that no monomials of $f$ are multiples of $x_{4} x_{5}$, so that

$$
f=g\left(x_{1}, x_{2}, x_{3}, x_{5}, x_{6}\right)+x_{4} h\left(x_{1}, x_{2}, x_{3}, x_{4}, x_{6}\right),
$$


for some homogeneous polynomials $g$ and $h$ of degrees $p$ and $(p-1)$, respectively. Consider the open subset $U$ of $Q_{4}$ given by $x_{5} \neq 0$. This subset is isomorphic to $\mathbb{C}^{4}$ with coordinates

$$
\left(y_{1}, \ldots, y_{4}\right)=\left(\frac{x_{1}}{x_{5}}, \frac{x_{2}}{x_{5}}, \frac{x_{3}}{x_{5}}, \frac{x_{6}}{x_{5}}\right)
$$

and the intersection of $D_{1}$ with $U$ is given by $y_{4}=0$. Every polynomial $f$ gives the polynomial on $U$

$$
\begin{aligned}
f\left(x_{1}, \ldots, x_{6}\right) x_{5}^{-p} & =f\left(y_{1}, y_{2}, y_{3}, y_{3} y_{4}, 1, y_{4}\right) \\
& =g\left(y_{1}, y_{2}, y_{3}, 1, y_{4}\right)+y_{3} y_{4} h\left(y_{1}, y_{2}, y_{3}, y_{3} y_{4}, y_{4}\right) .
\end{aligned}
$$

In order for this polynomial to vanish to order $(p-1)$ at $y_{4}=0$, it should be divisible by $y_{4}^{p-1}$. The total degrees of the monomials in $g$ are at most $p$, and the total degree of the monomials in $y_{3} y_{4} h$ are larger than $p$. Consequently, there will be no cancellations among these monomials, and each of them must be divisible by $y_{4}^{p-1}$. This means that $g=($ linear $) \cdot x_{6}^{p-1}$, and the total degree of all monomials in $h$ in $x_{4}$ and $x_{6}$ is at least $p-2$. This means that the total degree of all monomials in $f$ in $x_{4}$ and $x_{6}$ is at least $p-1$, which leads to (2.2).

Remark 2.6. If the variables $x_{1}$ and $x_{2}$ do not appear in (2.2), then the divisor $D$ will be a double cone over a $(1, p)$ curve $C \subset Q_{2}$. Divisors of this form play a crucial role in the study of orthogonal complex structures globally defined on $\mathbb{R}^{6}$, see [BSV].

When $p=3$, there is an irreducible subvariety of $Q_{6}$ of bidegree $(1,3)$ which does not lie in a $\mathbb{P}^{5} \subset \mathbb{P}^{7}$, and hence can not be of the type considered in Proposition 2.5. Geometrically, it can be described as follows. The intersection of the hyperplane $x_{8}=0$ with $Q_{6}$ given by (2.1) is a cone over the Grassmannian $G(2,4)$, which is identified with a nonsingular dimension four quadric in $\mathbb{P}^{5}$. Let us recall the Plücker equation for $G(2,4)$. If we have $w=\sum_{i<j} p_{i j} v_{i} \wedge v_{j}$, then the condition $w \wedge w=0$ means

$$
p_{12} p_{34}-p_{13} p_{24}+p_{23} p_{14}=0 \text {. }
$$

We will henceforth view $G(2,4)$ as a subset of $Q_{6}$ by the embedding

$$
\left(p_{12}, p_{13}, p_{23}, p_{14}, p_{24}, p_{34}\right) \mapsto\left(0, p_{12}, p_{13}, p_{23}, p_{14}, p_{24}, p_{34}, 0\right)
$$

There is a surface $S$ of type $(1,3)$ in $G(2,4)$ namely the quadratic Veronese embedding $\mathbb{P}^{2} \hookrightarrow G(2,4)$ given by

$$
\left(u_{0}: u_{1}: u_{2}\right) \mapsto\left(u_{0}^{2}: u_{0} u_{1}: u_{0} u_{2}: u_{1}^{2}-u_{0} u_{2}: u_{1} u_{2}: u_{2}^{2}\right) .
$$

The irreducible threefold of bidegree $(1,3)$ is then constructed as a cone over this surface, which is the image of the weighted projective space with variables $\left(u_{0}, u_{1}, u_{2}, u_{3}\right)$ with weights $(1,1,1,2)$ under the map given by

$$
\left(u_{0}: u_{1}: u_{2}: u_{3}\right) \mapsto\left(u_{3}: u_{0}^{2}: u_{0} u_{1}: u_{0} u_{2}: u_{1}^{2}-u_{0} u_{2}: u_{1} u_{2}: u_{2}^{2}: 0\right) .
$$


Indeed, by taking a cone over a surface of bidegree $(a, b)$ in $G(2,4)$ one gets a threefold of bidegree $(a, b)$ or $(b, a)$ in $Q_{6}$, so the variety (2.4) is of bidegree $(1,3)$ or $(3,1)$. Consider its intersection with the vertical $\mathbb{P}^{3}$ given by

$$
0=x_{1}=x_{3}=x_{5}=x_{7},
$$

which occurs at the single point $\left(u_{0}: u_{1}: u_{2}: u_{3}\right)=(1: 0: 0: 0)$. It is easy to verify that the intersection is transversal, therefore it must be of bidegree $(1,3)$.

We are now ready to state the main theorem.

Theorem 2.7. Every irreducible $Z$ of bidegree $(1, p)$ in $Q_{6}$ is given by one of the following, up to the action of $\operatorname{Aut}\left(Q_{6}\right)=\operatorname{PSO}(8, \mathbb{C})$.

- $p=0$ and $Z$ is a horizontal $\mathbb{P}^{3}$.

- $p=1$ and $Z$ is a smooth quadric in $\mathbb{P}^{4} \subset \mathbb{P}^{7}$.

- $p=3$ and $Z$ is the cone over the Veronese surface given by (2.4).

- $p \geq 1$ and $Z$ is the Weil divisor described in Proposition 2.5 in the rank four dimension four quadric $Q_{4} \subset Q_{6}$.

We will prove this theorem in Section 4. Meanwhile, we will make some remarks on this classification.

Remark 2.8. As a corollary of our classification, we see that any threefold of order one in $Q_{6}$ is contained in a $\mathbb{P}^{5}$, except in the third case (the span of the $Z$ in this case is a $\left.\mathbb{P}^{6}\right)$. Furthermore, in view of Proposition 2.5 , all but the second and third cases are characterized by the condition that span of $Z$ is contained in a $\mathbb{P}^{5} \subset \mathbb{P}^{7}$ such that the annihilator of this $\mathbb{P}^{5}$ with respect to $Q_{6}$ is an isotropic $\mathbb{P}^{1}$. This is because if $Z$ is a smooth quadric in a $\mathbb{P}^{4}$, then this $\mathbb{P}^{4}$ can not be embedded into a $\mathbb{P}^{5}$ with isotropic annihilator. Indeed, the annihilator of a $\mathbb{P}^{4}$ to which $Q$ restricts as a maximum rank quadric is a $\mathbb{P}^{2}$ with a maximum rank quadric, which does not contain any lines.

Remark 2.9. The automorphism group of $Q_{4}$ moves around the Weil divisors of type $(1, p)$ on it, and we have made no attempt to further restrict the shape of the equation $f$ in Proposition 2.5 to classify the orbits of this action. From the point of view of orthogonal complex structures, we are interested in classification of subvarieties of $Q_{6}$ up to the action of the real Lie group $\operatorname{SO}_{\circ}(7,1)$, which is the $\operatorname{subgroup~of~} \operatorname{PSO}(8, \mathbb{C})$ that preserves the twistor fibration $\mathbb{P}^{3} \rightarrow Q_{6} \rightarrow S_{6}$, see [BSV].

Using this classification, we can characterize exactly which threefolds of order one are smooth. The proof of the following theorem is found in Section 4 .

Theorem 2.10. There are only three smooth threefolds of order one in $Q_{6}$ up to $\operatorname{Aut}\left(Q_{6}\right)$, given in the following list.

- $p=0$ and $Z$ is a horizontal $\mathbb{P}^{3}$.

- $p=1$ and $Z$ is a smooth quadric in $\mathbb{P}^{4} \subset \mathbb{P}^{7}$.

- $p=2$ and $Z$ is the Segre embedding $\mathbb{P}^{1} \times \mathbb{P}^{2} \hookrightarrow \mathbb{P}^{5} \subset \mathbb{P}^{7}$ given by

$\left(\left(u_{0}: u_{1}\right),\left(v_{0}: v_{1}: v_{2}\right)\right) \mapsto\left(u_{0} v_{0}: u_{1} v_{0}: u_{0} v_{1}: u_{0} v_{2}: u_{1} v_{1}: u_{1} v_{2}: 0: 0\right)$,

which corresponds to $f=x_{1} x_{6}-x_{2} x_{4}$ in (2.2). 


\section{Preliminary Results on the geometry of $Q_{6}$ And $Z$}

Here we collect various facts that will be useful later. For the remainder of the paper we will assume that $Z$ is an irreducible threefold of bidegree $(1, p)$ in $Q_{6}$.

Proposition 3.1. If $Z$ has homology class $(1, p)$, then it has degree $p+1$ in $\mathbb{P}^{7}$.

Proof. Horizontal and vertical $\mathbb{P}^{3}$-s have bidegree $(1,0)$ and $(0,1)$ respectively and degree one. It remains to observe that degree is a linear function on $H_{6}\left(Q_{6}, \mathbb{Z}\right)$.

Proposition 3.2. The space of vertical (or horizontal) $\mathbb{P}^{3}-s$ in $Q_{6}$ is of dimension six, and is isomorphic to a nonsingular quadric. The space of vertical (or horizontal) $\mathbb{P}^{3}$-s through a given point in $Q_{6}$ is of dimension 3 and is isomorphic to a $\mathbb{P}^{3}$. The space of vertical (or horizontal) $\mathbb{P}^{3}$-s through a given $\mathbb{P}^{1} \subset Q_{6}$ is of dimension one and is isomorphic to a $\mathbb{P}^{1}$. There is exactly one vertical and one horizontal $\mathbb{P}^{3}$ through a given $\mathbb{P}^{2} \subset Q_{6}$.

Proof. This follows from the classical description of the isotropic Grassmannians of low dimension. The first space is described by oriented or reverse oriented isotropic 4-planes in $\mathbb{C}^{8}$, which are determined by a projective pure spinor. In dimension 8 , a pure spinor satisfies a single quadratic relation of rank 8, so these spaces are each nonsingular quadrics of dimension 6, see [Car81, Chapter VI]. For the last three spaces of the proposition, we need to consider oriented isotropic 4-planes in $\mathbb{C}^{8}$ containing a fixed line $P_{1}$, plane $P_{2}$, or 3-plane $P_{3}$, respectively. This is equivalent to choosing an oriented isotropic $(4-j)$-plane $V_{j} \subset \operatorname{Ann}\left(P_{j}\right) / P_{j}=\mathbb{C}^{2(4-j)}$. This space is a $\mathbb{P}^{3}$, $\mathbb{P}^{1}$, or a single point since all non-zero spinors are pure in dimensions 6,4 , and 2 , respectively, see [Car81, Chapter VI].

Lemma 3.3. If $p \neq 1$ and $Z$ is contained in $a \mathbb{P} \cong \mathbb{P}^{6} \subset \mathbb{P}^{7}$, then $Q_{6} \cap \mathbb{P}$ is singular.

Proof. Suppose $Q_{6} \cap \mathbb{P}$ is a smooth 5-quadric $Q_{5}$. By the Lefschetz hyperplane theorem and Poincarè duality, $H_{6}\left(Q_{5}, \mathbb{Z}\right) \cong \mathbb{Z}$, with the generator given by the intersection of 2 hyperplanes with $Q_{5}$. Consider the image of $H_{6}\left(Q_{5}, \mathbb{Z}\right)$ in $H_{6}\left(Q_{6}, \mathbb{Z}\right)$ under the inclusion map. The generator maps to the intersection of 3 hyperplanes with $Q_{6}$, which is $Q_{6} \cap \mathbb{P}^{4}$. We may choose the $\mathbb{P}^{4}$ so that the rank of the quadratic form drops to 2 , thus the generator will map to a 3-quadric of rank 2 which is the union of a horizontal $\mathbb{P}^{3}$ and a vertical $\mathbb{P}^{3}$ and is therefore of bidegree $(1,1)$. Consequently, the image of $H_{6}\left(Q_{5}, \mathbb{Z}\right)$ in $H_{6}\left(Q_{6}, \mathbb{Z}\right)$ consists of classes of type $(m, m)$. Since the map from $Z$ to $Q_{6}$ factors through $Q_{5}$, the class of $Z$ is of type $(m, m)$, so $p=1$, contradiction.

The following trick is one of the key technical tools of the paper.

Lemma 3.4. Let $Z$ be a dimension three subvariety of $Q_{6}$ of bidegree $(1, p)$. Any vertical $\mathbb{P}^{3}$ either intersects $Z$ transversely in a single point, or contains at least a one-dimensional set of points of $Z$.

Proof. Suppose that $Z$ intersects the vertical $\mathbb{P}^{3}$ at a disjoint set of points $\left\{z_{1}, \ldots, z_{k}\right\}$. Then the intersection of the corresponding homology classes equals to the sum of 
local contributions. On one hand, this intersection equals 1. On the other hand, every transveral intersection point contributes 1 and every non-transversal isolated intersection point contributes strictly more than 1, see [Ful98, Proposition 8.2 (a) and (c)].

A typical application of Lemma 3.4 is the following.

Lemma 3.5. If $A$ is a singular point of $Z$, then $Z \subset \operatorname{Ann}(A)$.

Proof. Consider the vertical $\mathbb{P}^{3}$-s that contain $A$. Each of them intersects $Z$ nontransversely at $A$, consequently, each of them must contain at least a dimension one subset of $Z$. There is a dimension three space of such $\mathbb{P}^{3}$-s, so the space of pairs

$$
W \equiv\left\{(P, x) \mid P \text { is a vertical } \mathbb{P}^{3} \text { containing } A \text {, and } x \in P \cap Z\right\},
$$

is at least four-dimensional. Consider the projection of this space to the second factor $\pi_{2}: W \rightarrow Z$, and observe that the fibers outside of $\operatorname{Ann}(A) \cap Z$ are empty. Fibers over points of $\operatorname{Ann}(A) \cap Z$ are one-dimensional by Proposition 3.2 . This shows that $\operatorname{Ann}(A) \cap Z$ must be three-dimensional, so $Z \subset \operatorname{Ann}(A)$ since $Z$ is irreducible.

The following proposition gives another restriction on the intersection of $Z$ with a vertical $\mathbb{P}^{3}$.

Proposition 3.6. If $Z$ intersects a vertical $\mathbb{P}^{3}$ in a set of dimension two, then this set must consist of a $\mathbb{P}^{2}$, and perhaps some lower dimensional irreducible components.

Proof. Denote this vertical $\mathbb{P}^{3}$ by $P$, and let $Z_{0}$ be the union of the 2-dimensional irreducible components of $P \cap Z$. Assume that the total degree of $Z_{0}$ is $d>1$. Consider the space $S$ of all vertical $\mathbb{P}^{3}-$ s in $Q_{6}$ which intersect $P$ in a line. By Proposition 3.2, this is a 5-dimensional manifold. Define

$$
W \equiv\left\{\left(P_{1}, z\right) \mid P_{1} \in S \text { and } z \in P_{1} \cap\left(Z \backslash Z_{0}\right)\right\}
$$

We claim that for generic $P_{1} \in S, \operatorname{dim}\left(P_{1} \cap\left(Z \backslash Z_{0}\right)\right) \geq 1$. Indeed, otherwise $P_{1}$ and $Z$ intersect at isolated points, provided we choose $P_{1}$ so that the corresponding line in $P$ intersects $Z_{0}$ in isolated points. We can account for $d$ points of intersection on $Z_{0}$, and all other points of intersection contribute positively, which contradicts the fact that $Z$ is of order one. This implies that $\operatorname{dim}(W) \geq 6$.

On the other hand, $W$ projects to $Z \backslash Z_{0}$, which is of dimension three. The fibers of this projection are of dimension at most two, since any $P_{1} \in S$ containing $z$ is completely determined by choice of a line in the two-dimensional subspace $\operatorname{Ann}(z) \cap P$. This implies that $\operatorname{dim}(W) \leq 5$, which is a contradiction. Consequently, if $Z_{0}$ is nonempty then it has degree one, which implies that it is a linear subspace GH94, page 174].

The following two lemmas describe the intersection of $Z$ with a horizontal $\mathbb{P}^{3}$.

Lemma 3.7. If $Z$ intersects a horizontal $\mathbb{P}^{3}$ (set-theoretically) at a set of dimension one, then this set must consist of one line in $\mathbb{P}^{3}$ and perhaps some disjoint points. 
Proof. Denote this horizontal $\mathbb{P}^{3}$ by $P$, and denote by $C$ the union of the dimension one components of $Z \cap P$. Define

$$
W^{\prime} \equiv\left\{\left(P^{\prime}, z\right) \mid P^{\prime} \text { is a vertical } \mathbb{P}^{3} \text { with } \operatorname{dim}\left(P^{\prime} \cap P\right)=2 \text {, and } z \in\left(Z \cap P^{\prime}\right) \backslash P\right\} \text {. }
$$

If the degree of $C$ (in $P=\mathbb{P}^{3}$ ) is strictly larger than one, it means that a generic $P^{\prime}$ as above intersects $C$ in at least two points, since the degree of a curve in $P$ is given by counting transverse intersections with a $\mathbb{P}^{2} \subset P$. By Lemma 3.4, a generic such $P^{\prime}$ contains at least a curve of points of $Z$. This means that the generic fiber of the projection on the first factor is at least 1 dimensional. By Proposition 3.2, there is a dimension three space of such $P^{\prime}$, so the dimension of $W^{\prime}$ is at least 4 . However, all fibers under the second projection are points. This follows because for $z \in Z \backslash P$, $\operatorname{dim}(\operatorname{Ann}(z) \cap P)=2$, so the fiber over $z$ is the vertical $\mathbb{P}^{3}$ given by $\operatorname{Span}(z, \operatorname{Ann}(z) \cap P)$. Since $\operatorname{dim} Z=3$, this implies that $\operatorname{dim}\left(W^{\prime}\right) \leq 3$, a contradiction.

Lemma 3.8. For $p>0$, the generic horizontal $\mathbb{P}^{3}$ intersects $Z$ transversely at $p$ disjoint smooth points.

Proof. Consider the space of pairs $(z, P)$ where $z \in Z$ and $P$ is a horizontal $\mathbb{P}^{3}$ that contains $z$. It is a $\mathbb{P}^{3}$-bundle over $Z$, so it is of dimension 6 . Consider the projection to the space of all horizontal $\mathbb{P}^{3}$. Since $p>0$, the fibers of this projection are nonempty. Since it is a map between projective varieties of the same dimension (six), it is generically finite. The generic fiber will consist of smooth points, which means that the generic horizontal $\mathbb{P}^{3}$, call it $H$, intersects $Z$ transversely at smooth points. By the definition of bidegree, $H$ will then necessarily intersect $Z$ at $p$ disjoint points.

The following construction will also be used in the proof of our main theorem.

Lemma 3.9. Let $H$ be a horizontal $\mathbb{P}^{3}$, and consider the blowup $\operatorname{Bl}\left(Q_{6}, H\right)$ of $Q_{6}$ along $H$. Then $\operatorname{Bl}\left(Q_{6}, H\right)$ is naturally identified with a bundle $\mathbb{P}^{3} \rightarrow B \stackrel{\pi}{\rightarrow} \mathbb{P}^{3}=H^{*}$, which is defined by specifying that a fiber of this bundle is the vertical $\mathbb{P}^{3}$ that intersects $H$ at a given $\mathbb{P}^{2} \in H^{*}$ (see Proposition 3.2). A point $z \notin H$ is mapped to the 2-plane Ann $(z) \cap H$. This map extends smoothly to the exceptional divisor, which is the projectivized normal bundle of $H$. The image of the exceptional divisor over a point $z \in H$ consists of all vertical 2-planes which intersect $H$ in a $\mathbb{P}^{2}$ containing $z$.

Proof. It is well-known that the blowup $\mathrm{Bl}\left(\mathbb{P}^{7}, H\right)$ of $\mathbb{P}^{7}$ at $H$ maps to $\mathbb{P}^{3}$ that parametrizes the $\mathbb{P}^{4}$-s in $\mathbb{P}^{7}$ that contain $H$. The blowup of $H$ in $Q_{6}$ is the proper preimage of $Q_{6}$ in $\operatorname{Bl}\left(\mathbb{P}^{7}, H\right)$, so it projects naturally to $\mathbb{P}^{3}$. Note that for each $\mathbb{P}^{4}$ that contains $H$, its intersection with $Q_{6}$ is the union of $H$ and a vertical $\mathbb{P}^{3}$ that intersects $H$ at a $\mathbb{P}^{2}$. The lemma follows easily from these facts.

\section{PRoOF OF THE MAIN THEOREMS}

Theorem 4.1. Let $Z$ be a subvariety of $Q_{6}$ of bidegree $(1, p)$. Let us further assume that $Z$ is singular or that $Z$ is smooth and $p \leq 3$. Then for $p \neq 3, Z$ is contained in

$a \mathbb{P}^{5}$. For $p=3$, either $Z$ is contained in a $\mathbb{P}^{5}$, or $Z$ is equivalent to the Veronese embedding (2.4) under an automorphism of $Q_{6}$. 
Proof. From Proposition 3.1, $\operatorname{deg}(Z)=p+1$, consequently, $Z$ is contained in a $\mathbb{P}^{p+3}$, see [GH94, page 174]. This implies the statement for $p<3$. For $p=3$ similarly $Z$ is contained in a $\mathbb{P}^{6}$. If $Z$ is singular then $Z$ is contained in a $\mathbb{P}^{6}$ by Lemma 3.5. In either case, by Lemma 3.3 , there is a point $A$ in $Q_{6}$ such that this $\mathbb{P}^{6}$ is $\operatorname{Ann}(A)$ and $Q_{6} \cap \mathbb{P}^{6}$ is a cone with vertex $A$ over the smooth quadric in $\mathbb{P}^{5}$, which we will identify with the Grassmannian $G(2,4)$. Consider the projection from $Z \backslash A$ to $G(2,4)$ from the point $A$.

First, consider the case when the image is a threefold $Y$ (if $A$ is contained in $Z$ then we consider the closure of the image of $Z \backslash A$ ). The divisor $Y$ is a zero set of a section of a line bundle $\mathcal{L}=\pi^{*} \mathcal{O}_{\mathbb{P}^{5}}(d)$ for some $d>0$, where $\pi: G(2,4) \rightarrow \mathbb{P}^{5}$ is the Plücker embedding. If $d>1$, for any smooth point $x \in Y$ consider the space

$$
W_{x} \equiv\left\{H=\mathbb{P}^{2} \subset G(2,4) \text { with } x \in H \text { and } \operatorname{Span}(H, A) \text { is a horizontal } \mathbb{P}^{3}\right\} \text {. }
$$

The span of the tangent spaces at $x$ of all such $H \in W_{x}$ is the entire $T(x, G(2,4))$. As a result, a generic $H$ will have a point $x \in H \cap Y$ such that $T(x, H)$ that does not lie in $T(x, Y)$, and thus $H$ and $Y$ will intersect transversely near $x$ inside $G(2,4)$. Consequently, the restriction to $H$ of the section of $\mathcal{L}$ whose zero set is $Y$, is smooth at $x \in H$. On the other hand, the pullback of $\mathcal{L}$ to $H$ is $\mathcal{O}_{H}(d)$, which shows that the set-theoretic intersection of $Y$ and $H$ is a (perhaps reducible) curve $C$ of total degree strictly larger than one. From transversality at $x$, we know that $Y$ does not intersect $H$ at a multiple of a single line, thus $C$ is not a line. Since $H$ is generic, we may assume that $P=\operatorname{Span}(H, A)$ intersects $Z$ at a curve $\tilde{C}$, rather than a surface (and perhaps some isolated points). Since the curve $\tilde{C}$ covers $C$ under projection, it cannot be a line either. This contradicts Lemma 3.7, therefore $d=1$ and $Y$ is contained in a $\mathbb{P}^{4}$, so $Z$ is contained in a $\mathbb{P}^{5}$ as claimed.

Otherwise, the image of $Z$ must be a surface $S$, and $Z$ must be a cone over that surface with vertex $A$. Since $Z$ is irreducible, this surface must be an irreducible surface of bidegree $(1, p)$ in $G(2,4)$. From [Ran86, $S$ is either of the form $S(M, F)$ which is by definition contained in the hyperplane in $\mathbb{P}^{5}$ determined by the line $M$, or $S$ is equivalent under an automorphism of $G(2,4)$ to the $(1,3)$ surface given by the secant line image of the twisted cubic. Under the Plücker embedding $G(2,4) \subset \mathbb{P}^{5}$, the image of this surface is the Veronese surface (2.3). To see this, the (affine part of the) rational normal cubic is the set of points $\left\{\left(1: t: t^{2}: t^{3}\right) \mid t \in \mathbb{C}\right\} \subset \mathbb{P}^{3}$, so we can parametrize a dense open subset of the surface $S$ by

$$
\left\{\left(1: t: t^{2}: t^{3}\right) \wedge\left(1: s: s^{2}: s^{3}\right):(s, t) \in \mathbb{C}^{2}\right\}
$$

which gives

$$
\begin{aligned}
\left(x_{2}: \ldots: x_{7}\right) & =\left(p_{12}: p_{13}: p_{23}: p_{14}: p_{24}: p_{34}\right) \\
& =\left(s-t: s^{2}-t^{2}: t s^{2}-s t^{2}: s^{3}-t^{3}: t s^{3}-s t^{3}: t^{2} s^{3}-s^{2} t^{3}\right) \\
& =\left(1: s+t: t s: s^{2}+s t+t^{2}: t s(s+t): t^{2} s^{2}\right) .
\end{aligned}
$$


This can then be parametrized in terms of the symmetric functions $\left(u_{1}, u_{2}\right)=(s+t, s t)$ as

$$
\left(1: u_{1}: u_{2}: u_{1}^{2}-u_{2}: u_{1} u_{2}: u_{2}^{2}\right)
$$

Finally, we homogenize to obtain (2.3) , and observe that any automorphism of $G(2,4)$ extends to an automorphism of $Q_{6}$ which maps $A$ to the point $(1: 0: \ldots: 0)$.

The following key result allows us to deal with smooth $Z$ for $p>3$.

Theorem 4.2. If $Z$ is a smooth subvariety in $Q_{6}$ of bidegree $(1, p)$ with $p>3$ then $Z$ is contained in a $\mathbb{P}^{5}$.

Proof. We take a generic $H$ as in Lemma 3.8, which intersects $Z$ at $p$ disjoint points, call these points $\left\{z_{1}, \ldots, z_{p}\right\}$. Consider $\operatorname{Bl}\left(Q_{6}, H\right)$, and the corresponding proper preimage $Y$ of $Z$. The blowup of $Q_{6}$ at $H$ induces a map $Y \rightarrow Z$ which is the blowup of $Z$ at the scheme-theoretic intersection of $H$ and $Z$. Since $H$ intersects $Z$ transversely, $Y \rightarrow Z$ is the blowup of $Z$ at the points $z_{i}$. The exceptional divisors $E_{i}$ of $Y \rightarrow Z$ are isomorphic to $\mathbb{P}^{2}$. Consider the restricted projection map $\pi: Y \rightarrow \mathbb{P}^{3}$. As noted in Lemma 3.9 , it maps each $E_{i}$ isomorphically to a $\mathbb{P}^{2}$ in $\mathbb{P}^{3}$ that could be identified with the space of vertical $\mathbb{P}^{3}$-s that intersect $H$ in a $\mathbb{P}^{2}$ containing $z_{i}$.

The fibers of $\pi$ are nonempty, so the generic fiber is smooth of dimension zero, so it is a point by Lemma 3.4. Consequently, $\pi: Y \rightarrow \mathbb{P}^{3}$ is a birational map of smooth varieties, which implies that its fibers are connected by Zariski's Main Theorem [Har77, Corollary III.11.4]. Consider the exceptional divisors of $\pi$ on $Y$. We claim that for each pair $(i, j), i \neq j$ there is an exceptional divisor $D_{i j}$ of $\pi$ which is contracted to the line $l_{i j}=\pi\left(E_{i}\right) \cap \pi\left(E_{j}\right)$. Indeed, for every point in $l_{i j}$ the corresponding vertical $\mathbb{P}^{3}$ that intersects $H$ at a $\mathbb{P}^{2}$ contains both $z_{i}$ and $z_{j}$. By Lemma 3.4, the fiber over this point is of dimension at least one. Because the fibers of $\pi$ are connected, for each $j \neq i$ there is an exceptional divisor $D_{i j}$ of $\pi$ which maps to $l_{i j}$ and intersects $E_{i}$ at a curve.

We next consider $K_{Y}$, the canonical class of $Y$. Since $\pi$ is a birational map, we have the equivalence

$$
K_{Y}=\pi^{*} K_{\mathbb{P}^{3}}+\sum_{r} a_{r} D_{r}
$$

for the exceptional divisors $D_{r}$ of $\pi$, where the discrepancies $a_{r}$ are the orders of vanishing of the Jacobian, and hence are positive integers. Since $E_{i}$ is obtained by blowing up a point on a smooth threefold, we have $K_{Y} \mid E_{i}=-2 l$ where $l$ is the class of a line on $E_{i}$. Restricting (4.1) to $E_{i}$, we then have

$$
-2 l=\left.K_{Y}\right|_{E_{i}}=-4 l+\left.\sum_{r} a_{r} D_{r}\right|_{E_{i}}
$$

This shows that the total degree of intersection of $D_{r}$ with $E_{i}$ is at most two. However, for all $j \neq i$, the divisors $D_{i j}$ intersect $E_{i}$, and the number of these divisors is at least $p-1>2$. This means that for each $i$ the number of distinct divisors $D_{i j}$ is at most two. As a consequence, the number of distinct lines $l_{i j}$ is at most two. This can be 
rephrased as saying that for each $i$ the number of distinct lines $\operatorname{Span}\left(z_{i}, z_{j}\right)$ in $H$ is at most two.

We claim that this means that all $z_{i}$ lie in a line. Indeed, consider the line that contains the maximum number of $z_{i}$. If it contains only two $z_{i}$, then there are strictly more than two lines through $z_{1}$, since $p>3$. Else, if there is a point $z_{j}$ not on this line, then there are at least three lines through that $z_{j}$. In either case, there is a point with at least 3 distinct lines passing through it, contradicting the conclusion from the preceeding paragraph. Denote the line containing all the $z_{i}$ by $L$.

Let us now consider the projection of $Z_{1}=Z-\cup_{i} z_{i}$ from the line $L$. That is, we let $W_{1} \cong \mathbb{P}^{5}$ denote the space of $\mathbb{P}^{2}$-s in $\mathbb{P}^{7}$ containing $L$, and to each point $x$ in $Z_{1}$ we associate the $\mathbb{P}^{2} \in W_{1}$ which is the span of $x$ and $L$. We claim that the dimension of the image of $Z_{1}$ is three. Indeed, the dimension of the image is at most three. If the dimension is strictly less than three, then a generic $\mathbb{P}^{2} \in W_{1}$, call it $Q$, does not intersect the image of $Z_{1}$. The subspace $Q$ is a $\mathbb{P}^{2}$-family of $\mathbb{P}^{2}$-s, each of which contains $L$. The image of $Z_{1}$ under the projection is all the $\mathbb{P}^{2}$-s containing $L$ which hit a point of $Z_{1}$. This means that each $\mathbb{P}^{2}$ in our $\mathbb{P}^{2}$-family of $\mathbb{P}^{2}$-s does not hit any point of $Z_{1}$. The union of all of these $\mathbb{P}^{2}$-s is a $\mathbb{P}^{4}$. This $\mathbb{P}^{4}$ therefore only hits $Z$ on $L$, that is, this $\mathbb{P}^{4}$ hits $Z$ exactly at the points $z_{i}$, with transverse intersection since $Q$ is generic. This shows that the degree of $Z$ in $\mathbb{P}^{7}$ is $p$. However, this degree is $p+1$, see Proposition 3.1. This contradiction proves that the image of $Z_{1}$ is of dimension three.

Next, we claim that the degree of the closure of the image of $Z_{1}$ is one. We choose a $\mathbb{P}^{2} \in W_{1}$, call it $Q$, which hits $\operatorname{Im}\left(Z_{1}\right)$ transversely in $k$ points, where $k$ is the degree of $\overline{\operatorname{Im}\left(Z_{1}\right)} \subset \mathbb{P}^{5}$. This means that the number of $\mathbb{P}^{2}$-s in $Q$ which intersect $Z_{1}$ is exactly $k$. The corresponding $\mathbb{P}^{4} \subset \mathbb{P}^{7}$ then intersects $Z$ in $p+r k$ points, where $r$ is the degree of the projection map. We must therefore have $p+r k=p+1$, which implies that $k=1$. Consequently, $\overline{\operatorname{Im}\left(Z_{1}\right)}$ is a $\mathbb{P}^{3}$ in $W_{1}$, which implies that $Z$ is contained in the corresponding $\mathbb{P}^{5}$ in $\mathbb{P}^{7}$.

Remark 4.3. In the proof of Theorem 2.10 below, we will see that the case considered above in Theorem 4.2 does not actually occur. Furthermore, it would not be difficult to extend the above proof to cover the singular case. However, it is more convenient to quote the result from [Ran86] to deal with this case, as we have done in the proof of Theorem 4.1 above.

We are now ready to prove our main results.

Proof of Theorem 2.7. By Theorems 4.1 and 4.2, the variety $Z$ is either given by (2.4) or is contained inside a $\mathbb{P}^{5} \subset \mathbb{P}^{7}$. If $p=1$, then it is contained in a $\mathbb{P}^{4}$ in $\mathbb{P}^{7}$. This $\mathbb{P}^{4}$ intersects $Q_{6}$ in a dimension three quadric of rank at least two. However, rank two is not possible since this intersection is the union of a horizontal and a vertical $\mathbb{P}^{3}$, which is reducible. The rank could then be anywhere from 3 to 5 . When the rank is three or four, the annihilator of $\operatorname{Span}(Z)$ contains an isotropic line, in which case $Z$ can be moved inside $Q_{4}$ by an automorphism of $Q_{6}$, since the automorphism group 
acts transitively on isotropic lines. If the rank is five, than $Z$ is of the second case listed in Theorem 2.7.

If $p \neq 1$ and $Z$ is contained inside a $P \cong \mathbb{P}^{5} \subset \mathbb{P}^{7}$, then $Q_{6} \cap P$ is a singular quadric of rank 4. To see this, the rank of the quadratic form restricted to $P$ can be either 4,5 , or 6 . If the rank is 5 or 6 , then consider $\operatorname{Ann}(P) \cong \mathbb{P}^{1}$. Rank 4 is equivalent to $\operatorname{Ann}(P) \subset Q_{6}$, so choose a point $x \in \operatorname{Ann}(P) \backslash Q_{6}$. For this point $x$, $Z \subset \operatorname{Ann}(x) \cong \mathbb{P}^{6}$, and $\operatorname{Ann}(x) \cap Q_{6}$ is smooth, which contradicts Lemma 3.3. Noting that all singular quadrics of rank four and dimension four in $Q_{6}$ can be moved to $Q_{4}$ by an automorphism of $Q_{6}$, the theorem follows from Proposition 2.5.

We next find exactly which threefolds of order one are smooth. The proof amounts to directly verifying which divisors in Proposition 2.5 are smooth.

Proof of Theorem 2.10. For $p=0$ the statement is clear. For $p=1$ the only possibilities for $Z$ are the quadrics of ranks 3,4 and 5, of which only the quadrics of rank 5 are smooth. All rank 5 quadrics in $Q_{6}$ are equivalent under the action of $\operatorname{Aut}\left(Q_{6}\right)$. The third case in Theorem 2.7 is singular at the cone point. Therefore we need only consider the last case in Theorem 2.7.

We first consider the case $p>2$. We may assume that $Q_{4}$ is given by

$$
Q_{4}=\left\{x \in \mathbb{P}^{7} \mid x_{7}=x_{8}=0, x_{3} x_{6}=x_{4} x_{5}\right\},
$$

and by Proposition 2.5 that $Z$ is a Weil divisor on $Q_{4}$ such that $Z+(p-1) D_{1}$ is given by

$$
0=g_{p}\left(x_{4}, x_{6}\right)+\sum_{i=1,2,3,5} x_{i} g_{p-1}^{(i)}\left(x_{4}, x_{6}\right) .
$$

For any $(a, b) \in \mathbb{C}^{2} \backslash(0,0)$ consider the $\mathbb{P}^{3} \subset Q_{4}$ given by

$$
P_{(a, b)}=\left\{\left(u_{1}: u_{2}: u_{3} a: u_{4} a: u_{3} b: u_{4} b\right) \mid\left(u_{1}: u_{2}: u_{3}: u_{4}\right) \in \mathbb{P}^{3}\right\}
$$

which contains the singular line of $Q_{4}$

$$
L=\left\{\left(u_{1}: u_{2}: 0: \ldots: 0\right) \mid\left(u_{1}: u_{2}\right) \in \mathbb{P}^{1}\right\} .
$$

Clearly, $P_{(a, b)}$ depends only upon the class $(a: b) \in \mathbb{P}^{1}$. The Weil divisor $Z$ of class $(1, p)$ is a Cartier divisor on $Q_{4} \backslash L$, hence it restricts to a Cartier divisor on $P_{(a, b)}^{*} \equiv$ $P_{(a, b)} \backslash L$. The groups of Cartier divisors on $P_{(a, b)}$ and $P_{(a, b)}^{*}$ are isomorphic, and it is easy to see that the class of $Z$ when restricted to $P_{(a, b)}^{*}$ is $\mathcal{O}(1)$.

Explicitly, the equation (4.2) when restricted to $P_{(a, b)}$ can be written

$$
0=u_{4}^{p-1}\left(g_{p-1}^{(1)}(a, b) u_{1}+g_{p-1}^{(2)}(a, b) u_{2}+c_{3} u_{3}+c_{4} u_{4}\right)
$$

where $c_{3}$ and $c_{4}$ are constants. The factor $u_{4}^{p-1}$ amounts to the restriction of $D_{1}$ to $P_{(a, b)}^{*}$, so $Z \cap P_{(a, b)}^{*}$ is given by the second, linear, factor. Since $Z$ is irreducible, it can never contain a $\mathbb{P}^{3}$. Hence,

$$
Q_{(a, b)}=\overline{Z \cap P_{(a, b)}^{*}}
$$


is a $\mathbb{P}^{2}$ contained in $Z$. This $\mathbb{P}^{2}$ contains $L$ if and only if both $g_{p-1}^{(1)}(a, b)$ and $g_{p-1}^{(2)}(a, b)$ are zero. If they are not both zero, then $Q_{(a, b)}$ passes through a unique point on $L$ given by

$$
Q_{(a, b)} \cap L=\left(-g_{p-1}^{(2)}(a, b): g_{p-1}^{(1)}(a, b): 0: 0: 0: 0\right) .
$$

We are now ready for the main argument. View $g_{p-1}^{(1)}$ and $g_{p-1}^{(2)}$ as sections of $\mathcal{O}(p-1)$ on $\mathbb{P}^{1}$. If $g_{p-1}^{(1)}$ and $g_{p-1}^{(2)}$ have no common zeroes, then we can define the map $\psi: \mathbb{P}^{1} \rightarrow L$ by

$$
\psi((a: b))=\left(-g_{p-1}^{(2)}(a, b): g_{p-1}^{(1)}(a, b): 0: 0: 0: 0\right) .
$$

The map $\psi$ has degree $p-1$, and since $p-1>1$, generically every value has at least two preimages. Consequently, for a generic point $z \in L$, there exist two distinct $\mathbb{P}^{3}$-s as above, $P_{1}=P_{\left(a_{1}, b_{1}\right)}$ and $P_{2}=P_{\left(a_{2}, b_{2}\right)}$, such that $z \in Q_{\left(a_{1}, b_{1}\right)}$ and $z \in Q_{\left(a_{2}, b_{2}\right)}$. Since $P_{1} \cap P_{2}=L$, the corresponding $\mathbb{P}^{2}$-s in (4.4) intersect transversely. If $Z$ were smooth at $z$ then $T_{z} Z$, the tangent space to $Z$ at $z$, would contain both planes, so its dimension would be at least four. This contradiction shows that $Z$ is singular at $z$.

We next consider the cases where the map $\psi$ is not well-defined. If $g_{p-1}^{(1)}$ and $g_{p-1}^{(2)}$ are both zero, then $Z$ is a double cone over a $(1, p)$ curve in a smooth 2 -quadric (see Remark 2.6), which is singular for $p \geq 1$. There remains the case that $g_{p-1}^{(1)}$ and $g_{p-1}^{(2)}$ have a common root but are not both zero. Denote this common root by $\left(a_{0}, b_{0}\right)$. Consider the $\mathbb{P}^{3}$ corresponding to this root, $P_{\left(a_{0}, b_{0}\right)}$, and another $\mathbb{P}^{3}=P_{(a, b)}$ for some generic $\left(a_{1}, b_{1}\right)$ which is different from this root and has one of $g_{p-1}^{(1)}$ and $g_{p-1}^{(2)}$ nonzero. Denote by $z$ the intersection point of $Q_{\left(a_{1}, b_{1}\right)}$ and $L$ given in (4.5). Since $P_{\left(a_{0}, b_{0}\right)}$ contains $L$, it also passes through $z$. We have found two $\mathbb{P}^{2}$-s contained in $Z$ and passing through the point $z$. We again argue that they are transversal. Indeed, the intersection of $P_{\left(a_{0}, b_{0}\right)}$ and $P_{\left(a_{1}, b_{1}\right)}$ is $L$, so the intersection of the two $\mathbb{P}^{2}$-s is contained in $L$. However, $L$ is not contained in the second $\mathbb{P}^{2}$, hence the two $\mathbb{P}^{2}$-s through $z$ intersect at $z$ only. As above, this gives a contradiction. This finishes the proof that $Z$ is singular for $p>2$.

In the $p=2$ case, we can argue as in the preceeding paragraph to assume that $g_{1}^{(1)}$ and $g_{1}^{(2)}$ are both nonzero and have no common zeroes. By a linear change of $x_{1}$ and $x_{2}$ (which easily extends to an automorphism of $Q_{6}$ by an appropriate linear change of $x_{7}$ and $x_{8}$ ) we may assume that the equation (4.2) has the form

$$
0=x_{1} x_{6}-x_{2} x_{4}-x_{4} h_{2}\left(x_{3}, x_{4}, x_{5}, x_{6}\right)+x_{6} h_{1}\left(x_{3}, x_{4}, x_{5}, x_{6}\right),
$$

where $h_{1}$ and $h_{2}$ are linear. We claim that $h_{i}$ can be absorbed into $x_{i}$ by a linear change of coordinates which keeps $Q_{4}$ and $Q_{6}$ unchanged. If $h_{1}=a_{3} x_{3}+a_{4} x_{4}+a_{5} x_{5}+a_{6} x_{6}$, then we can use

$$
\left(x_{1}, \ldots, x_{8}\right) \rightarrow\left(x_{1}-a_{3} x_{3}-a_{5} x_{5}, x_{2}, x_{3}, x_{4}-a_{5} x_{8}, x_{5}, x_{6}+a_{3} x_{8}, x_{7}, x_{8}\right),
$$

and similarly for $h_{2}$ to reduce (4.7) on $Q_{4}$ to

$$
0=x_{1} x_{6}-x_{2} x_{4}-x_{4} \tilde{h}_{2}\left(x_{4}, x_{6}\right)+x_{6} \tilde{h}_{1}\left(x_{4}, x_{6}\right),
$$


and then use transformations analogous to (4.8) to eliminate all of the extra terms. This case is finished by observing that the equation $x_{1} x_{6}-x_{2} x_{4}=0$ in $Q_{4}$ cuts out $D_{1}$ and the image of the Segre embedding, so $Z$ is as claimed.

The technique in the above proof yields a geometric description of the last case in Theorem 2.7.

Proposition 4.4. If $Z$ is in the last case in Theorem 2.7, then $Z$ contains $L$ and is equal to the union of the $Q_{(a, b)}$ over all $(a: b) \in \mathbb{P}^{1}$. This is almost a disjoint union, in the sense that the only intersections occur at points of L. Furthermore, $Z$ is smooth away from $L$.

Proof. Either at least one of the $Q_{(a, b)}$ contains $L$ or all the points of $L$ have a $Q_{(a, b)}$ through it, so the union of $Q_{(a, b)}$ contains $L$. The complement of $L$ in $Q_{4}$ is the (disjoint) union of $P_{(a, b)}^{*}$, and each of them intersects $Z$ in an open subset of the corresponding $Q_{(a, b)}$. This shows that $Z$ is equal to the union of $Q_{(a, b)}$, and clearly the only intersections occur at points of $L$. Finally, if $Z$ were singular at some point $A$ outside of $L$, then by Lemma 3.5, $Z$ would be contained in $\operatorname{Ann}(A) \cap Q_{4}$, which is just a union of a vertical and a horizontal $\mathbb{P}^{3}$.

Remark 4.5. The above proof of Theorem 2.10 also leads to a criterion for when the polynomial $f$ in (2.2) leads to an irreducible divisor of bidegree $(1, p)$. If the corresponding $Z$ is reducible, then the $(1, p)$ Weyl divisor has to split up as a $\left(1, p_{1}\right)$ and some $(0,1)$ divisors, which are necessarily vertical subspaces. Therefore the condition that all $Q_{(a, b)}$ are $\mathbb{P}^{2}$-s is both necessary and sufficient.

\section{REFERENCES}

[BSV] Lev Borisov, Simon Salamon, and Jeff Viaclovsky, Orthogonal complex structures in Euclidean spaces, preprint, 2008.

[Car81] Élie Cartan, The theory of spinors, Dover Publications Inc., New York, 1981, A reprint of the 1966 English translation.

[Ful98] William Fulton, Intersection theory, second ed., Ergebnisse der Mathematik und ihrer Grenzgebiete. 3. Folge. A Series of Modern Surveys in Mathematics, vol. 2, Springer-Verlag, Berlin, 1998.

[GH94] Phillip Griffiths and Joseph Harris, Principles of algebraic geometry, Wiley Classics Library, John Wiley \& Sons Inc., New York, 1994, Reprint of the 1978 original.

[Gro92] Mark Gross, The distribution of bidegrees of smooth surfaces in $\operatorname{Gr}\left(1, \mathbf{P}^{3}\right)$, Math. Ann. 292 (1992), no. 1, 127-147.

[Har77] Robin Hartshorne, Algebraic geometry, Springer-Verlag, New York, 1977, Graduate Texts in Mathematics, No. 52.

[Ran86] Ziv Ran, Surfaces of order 1 in Grassmannians, J. Reine Angew. Math. 368 (1986), 119126.

Department of Mathematics, University of Wisconsin, Madison, Wi, 53706, USA

E-mail address: borisov@math.wisc.edu, jeffv@math.wisc.edu 